\title{
Detection of Biofilm Phenotype of Isolated Staphylococcus epidermidis from Respiratory Catheters of Hospitalized Patients and Evaluation the Effect of Antibodies against SesC Protein on Biofilm Formation
}

\author{
Shahla Samei Fard ${ }^{1 \#, ~ M a n s o u r ~ A m i n ~}{ }^{1 *}$, Laleh Khodaparast ${ }^{2 \#}$, Ladan Khodaparast ${ }^{2 \#}$, Parastoo Moradi Choghakabodi ${ }^{1 \#}$ and Mohammad Shahrooei $^{2 \#}$
}

${ }^{1}$ Department of Microbiology, School of Medicine, Ahvaz Jundishapur University of Medical Sciences, Ahvaz, Iran

${ }^{2}$ Department of Microbiology and Immunology, Laboratory of Clinical Bacteriology and Mycology, KU Leuven-University of Leuven, B-3000 Leuven, Belgium

"Corresponding author: Mansour Amin, Health Research Institute, Infectious and Tropical Diseases Research Center and Department of Microbiology, School of Medicine, Ahvaz Jundishapur University of Medical Sciences, Ahvaz, Iran, Tel: 00989381939339; E-mail: mnsamin@yahoo.com

\#Authors contributed equally

Received date: July 24, 2015; Accepted date: September 28, 2015; Published date: October 05, 2015

Copyright: $\odot 2015$ Fard SS, et al. This is an open-access article distributed under the terms of the Creative Commons Attribution License, which permits unrestricted use, distribution, and reproduction in any medium, provided the original author and source are credited.

\begin{abstract}
Staphylococcus epidermidis account for the majority of foreign body-related infections particularly catheter-related infections. Its ability to adhere to materials and to promote formation of a biofilm is the most important feature of its pathogenicity. The presence of $S$. epidermidis surface components particularly SesC protein is essential for biofilm formation. Accordingly, in addition to antibiotic therapy has recently been a lot of attention on the development of vaccines against $S$. epidermidis surface proteins. The aim of this study is detection of biofilm phenotype of isolated $S$. epidermidis from respiratory catheters of hospitalized patients and evaluation of the effect of antibodies against SesC protein on biofilm formation. In this study, we've isolated 70 coagulase-negative staphylococcus strains from respiratory catheter samples $(n=350)$. Then, 40 isolates of these strains were randomly selected; Twenty $(50 \%) S$. epidermidis strains were identified by Mass spectrometry from all 40 isolates. From these twenty strains, $30 \%$ produced dissolvable PIA-dependent biofilms in sodium metaperiodate after growth in Tryptic soy broth (TSB) with $\mathrm{NaCl}$ and $40 \%$ produced dissolvable protein-dependent biofilms in proteinase $\mathrm{K}$ after growth in TSB with glucose. Evaluated the effect of anti-SesC antibodies on biofilm formation by using a semi quantitative adherence assay. In $40 \%$ of cases, anti-SesC antibodies had significant inhibitory effect on biofilm formation $(P<0.05)$ in particular on protein-dependent biofilms. Although, the exact role of $\mathrm{SesC}$ in biofilm formation still is unknown but our findings display the importance of SesC protein on developing of biofilm formation of respiratory catheters.
\end{abstract}

Keywords: Staphylococcus epidermidis, Biofilm; SesC protein; Respiratory catheters

\section{Introduction}

Staphylococcus epidermidis is considered to be the major cause of device-related infections, especially catheter-related infections. These infections have increased in number, owing to the increased use of such devices [1]. The incidence of infection due to the use of medical devices is often dependent on the ability of bacteria to produce a hard multi-layer structure called "biofilm" on the surface of medical devices used to treat [2]. The main problem of microbial biofilm infections is tending to resist against clearing by the host immune system, as well as all antimicrobial agents [3]. Currently, the only completely effective method for curing biofilm infections is to remove the infected device, which is a risky, costly, and stressful procedure. Different strategies are used against biofilm infections, such as administration of bactericidal agents, modification of biomaterial surface to prevent initiation of bacterial colonization $[4,5]$. Although there is no certain cure for the prevention and treatment of biofilms, besides the use of antibiotics, a lot of attention recently is on the possibility of strengthening the immune system through vaccination against superficial factors such as polysaccharides and proteins involved in biofilm structure. Several recent studies have shown that antibodies against cell surface components of $S$. epidermidis can affect the rate of biofilm formation or adherence of these bacteria to medical devices in vitro [6]. Using an in silico procedure, identified 64 proteins that are predicted to be $S$. epidermidis surface exposed (Ses), and 2 of the largest $\mathrm{ABC}$ transporters (SesK and SesM)-were selected for evaluation as vaccine candidates. Anti-SesC antibodies exhibited the greatest inhibitory effect on $S$. epidermidis biofilm formation in vitro and on colonization and infection in a mouse jugular vein catheter infection model that includes biofilms and organ infections. Antibodies to SesC were shown to be opsonic by an in vitro opsonophagocytosis assay [7]. In the present study, Staphylococcus epidermidis strains were isolated from respiratory catheters samples used in hospitalized patients by differential biochemical tests and mass spectrometry [8]. Then the presence of SesC gene of all isolates was checked using PCR screening. After evaluating the ability of biofilm formation, the biofilm phenotype of isolated $S$. epidermidis was determined by using a semi quantitative adherence assay in 96-well polystyrene microtiter plates (BD Biosciences, Heidelberg, Germany)[9]. Finally, the effect of specific antibodies against SesC proteins on preventing the biofilm formation was evaluated by these strains in polystyrene microtiter plates.

\section{Materials and Methods}

\section{Bacterial isolates and species identification}

In this study, a total of 350 different respiratory catheter specimens (at different ages) from hospitalized patients from Ahvaz Jundishapur University of Medical Sciences were collected (hospitals Golestan, 
Citation: Fard SS, Amin M, Khodaparast L, Khodaparast L, Choghakabodi PM, et al. (2015) Detection of Biofilm Phenotype of Isolated Staphylococcus epidermidis from Respiratory Catheters of Hospitalized Patients and Evaluation the Effect of Antibodies against SesC Protein on Biofilm Formation. Clin Microbiol 4: 221. doi:10.4172/2327-5073.1000221

Page 2 of 6

Imam Khomeini, Sina, Razi). The materials of these respiratory catheter or endotracheal tubes were silicone from Nanjing Hong An Medical Appliance Co., Ltd. The isolates were inoculated at mannitol salt agar (MSA; Merck) and blood agar (BA, Merck) medium in order to separate $S$. epidermidis strains (incubated at $37^{\circ} \mathrm{C}$ and $24 \mathrm{~h}$ ). After the bacterial growth on MSA, were carried out, respectively, Gram stain, direct microscopic examinations and differential biochemical tests including Hemolysis on BA Plates, Tube-Coagulase test and also Novobiocin susceptibility test for determining $S$. epidermidis strains.

A total of 70 coagulase-negative staphylococcus strains were isolated from respiratory catheter samples $(n=350)$. Thereafter, 40 isolates of these strains were randomly selected; Twenty (50\%) $S$. epidermidis strains were identified by Mass spectrometry from all 40 isolates. Then, These strains were tested for antibiogram by using the VITEK $^{\circ} 2$ identification system (BioMérieux), for listed antibiotics: Benzylpenicillin $(10 \mu \mathrm{g})$, Oxacillin $(5 \mu \mathrm{g})$, Gentamicin $(10 \mu \mathrm{g})$, Kanamycin $(5 \mu \mathrm{g})$, Tobramycin $(5 \mu \mathrm{g})$, Levofloxacin $(10 \mu \mathrm{g})$ Moxifloxacin $(10 \mu \mathrm{g})$, Fusidic acid $(10 \mu \mathrm{g})$, Mupirocin $(10 \mu \mathrm{g})$, Clindamycin $(2 \mu \mathrm{g})$, Linezolid $(10 \mu \mathrm{g})$, Teicoplanin $(5 \mu \mathrm{g})$,Vancomycin $(10 \mu \mathrm{g})$, Minocycline $(10 \mu \mathrm{g})$, Tigecycline $(5 \mu \mathrm{g})$, Nitrofurantoin $(5 \mu \mathrm{g})$, Rifampicin $(10 \mu \mathrm{g})$, Trimethoprim $(5 \mu \mathrm{g})$; (Table 1).

\begin{tabular}{|c|c|c|}
\hline Antibiotics & Resistance & Sensetive \\
\hline FOX & 75 & 25 \\
\hline B-PEN & 98 & 3 \\
\hline OXA & 80 & 20 \\
\hline GEN & 53 & 48 \\
\hline KAN & 63 & 38 \\
\hline TOB & 53 & 48 \\
\hline LVX & 33 & 68 \\
\hline MXF & 33 & 68 \\
\hline ERY & 70 & 30 \\
\hline CLI & 63 & 38 \\
\hline LZD & 0 & 100 \\
\hline TEC & 0 & 100 \\
\hline VAN & 0 & 100 \\
\hline MIN & 10 & 90 \\
\hline TGC & 0 & 100 \\
\hline NIT & 0 & 100 \\
\hline FUS & 5 & 95 \\
\hline MUP & 20 & 80 \\
\hline RIF & 25 & 75 \\
\hline TMP & 33 & 68 \\
\hline
\end{tabular}

Table 1: The resistance rate to antibiotics for 40 staphylococci.

\section{PCR screening of SesC genes in clinical isolates}

The presence of SesC gene in $S$. epidermidis strains was checked using PCR amilifaction of sesC, as previously explained by Shahrooei et al. [6]. The primers used for PCR screening of SesC genes were SesR1 and SesF1 sets that were designed by Shahrooei et al. [6] for 388 bp fragment and SesC sequence.

\section{SesC-SF ...........GTTGATAACCGTCAACAAGG \\ SesC-SR ...........CATGTTGATCTTTTGAATCCC}

The RP62A S. epidermidis strain was used as positive control and distilled water (DW) as negative control. For each strain, genomic DNA was extracted using a QIAamp DNA minikit (Qiagen) with the addition of $30 \mu \mathrm{g}$ of lysostaphin/ml at the lysis step $[6,10]$.

\section{In vitro biofilm formation assays}

The Biofilm formation of $S$. epidermidis isolates were done using a semi-quantitative adherence assay in 96-well polystyrene microtiter plates (BD Biosciences, Heidelberg, Germany) as previously described [9]. Briefly, $20 \mu \mathrm{l}$ of frozen cultures of $S$. epidermidis strains was inoculated into $5 \mathrm{ml} \mathrm{TSB}$ medium and grown to the late-exponential/ stationary-growth phase in a shaking incubator at $37^{\circ} \mathrm{C}$. Cultures were subsequently diluted in TSB to an OD600 of $0.005\left(5 \times 10^{6} \mathrm{CFU} / \mathrm{ml}\right)$ in fresh TSB. Then, $200 \mu$ portions of the mixtures were pipetted into $96-$ well polystyrene microtiter plates (BD Biosciences, Heidelberg, Germany), followed by incubation for $24 \mathrm{~h}$ at $37^{\circ} \mathrm{C}$ with no substance; were used three wells for each of strain. After the incubation, the plates were washed three times with phosphate-buffered saline (PBS $[\mathrm{pH}$ 6.8 ], containing $0.5 \mathrm{M} \mathrm{NaCl}$ and $10 \mathrm{mM}$ EDTA), and adherent biofilms were stained with $200 \mu \mathrm{l}$ of $1 \%$ (wt/vol) crystal violet (Sigma) for 10 min, after which the plates were washed three times with water and dried. For quantification, $160 \mu \mathrm{l}$ of $30 \%$ (vol/vol) acetic acid was added to each well to dissolve the stain. The OD595 of the dissolved stain was measured in a multipurpose UV/VIS plate reader. $S$. epidermidis strain $10 \mathrm{~b}$ in TSB was used as positive control, and TSB without bacteria was used as a negative control $[6,10]$.

\section{In vitro assay for determination of biofilm-forming types of bacteria}

After determining the biofilm forming isolates in TSB, the effect of addition of $\mathrm{NaCl}(4 \% \mathrm{NaCl})$, glucose (1\% glucose) to medium (TSB) or sodium metaperiodate $(\mathrm{SM})$ and proteinase $\mathrm{K}(\mathrm{PK})$ on biofilms of isolates formed in TSB with $1 \%$ glucose, in microtiter plates, after $24 \mathrm{~h}$ incubation at $37^{\circ} \mathrm{C}$ were assessed. This test was carried out as same as previous test using a semi-quantitative adherence assay. Briefly, $10 \mu \mathrm{l}$ bacterial suspension diluted in TSB to an OD600 of $0.005\left(5 \times 10^{6}\right.$ $\mathrm{CFU} / \mathrm{ml}$ ) in fresh TSB with $1 \%$ glucose, were pipetted into sterile 96 well polystyrene microtiter plates and statically incubated overnight at $37^{\circ} \mathrm{C}$. After $24 \mathrm{~h}$ incubation, the growth medium was replaced with 200 $\mu \mathrm{SM}$ buffer (10 mM SM, $50 \mathrm{mM}$ sodium acetate) or PK buffer [1 $\mathrm{mg} / \mathrm{ml}$ in $20 \mathrm{mM}$ Tris/HCl (pH7.5), $100 \mathrm{mM} \mathrm{NaCl}$ ]. Subsequently, plates were incubated at $37^{\circ} \mathrm{C}$ for $2 \mathrm{~h}$ and the remaining biofilms were quantified as explained above $[9,11]$.

\section{In vitro biofilm inhibition assays}

The effect of specific anti-SesC antibodies ( $\alpha$ SesC-IgGs) produced as earlier described [10] on in vitro biofilm formation during overnight (accumulation and establishment phase) was assessed using a semi- 
Citation: Fard SS, Amin M, Khodaparast L, Khodaparast L, Choghakabodi PM, et al. (2015) Detection of Biofilm Phenotype of Isolated Staphylococcus epidermidis from Respiratory Catheters of Hospitalized Patients and Evaluation the Effect of Antibodies against SesC Protein on Biofilm Formation. Clin Microbiol 4: 221. doi:10.4172/2327-5073.1000221

Page 3 of 6

quantitative adherence assay [6]. In first instance, $1 \times 10^{6}$ bacteria were incubated with $a \mathrm{SesC}$-IgGs $(20 \mu \mathrm{g} / \mathrm{ml}$ bacterial suspension $)$ for $2 \mathrm{~h}$ at $4^{\circ} \mathrm{C}$. Plates were then placed without shaking overnight at $37^{\circ} \mathrm{C}$ to allow bacterial growth and biofilm formation. The OD595 of dissolved stain was measured in a multipurpose UV/VIS plate reader.

\section{Statistical analysis}

A significant difference in biofilm formation in the presence of specific anti-SesC antibodies ( $\alpha$ SesC-IgGs) was tested with a TWO Way ANOVA analysis $[6,10]$.

\section{Results}

In this study, 70 CoNS strains were isolated. Then, 40 isolates of these strains were randomly selected; Twenty (50\%) S. epidermidis strains were identified by mass spectrometry from all 40 isolates. All $S$. epidermidis strains $(\mathrm{n}=20)$ resistant against to Benzyl-Penicillin and susceptible to Vancomycin, Tigecycline and Nitrofurantoin antibiotics (Table. 2).

\section{Presence of SesC gene in S. epidermidis isolates}

All 20 isolates identified as S. epidemidis out of 40 CoNS were sesC positive (Figure 1), as it has been previously reported by Shahrooei et al [6].

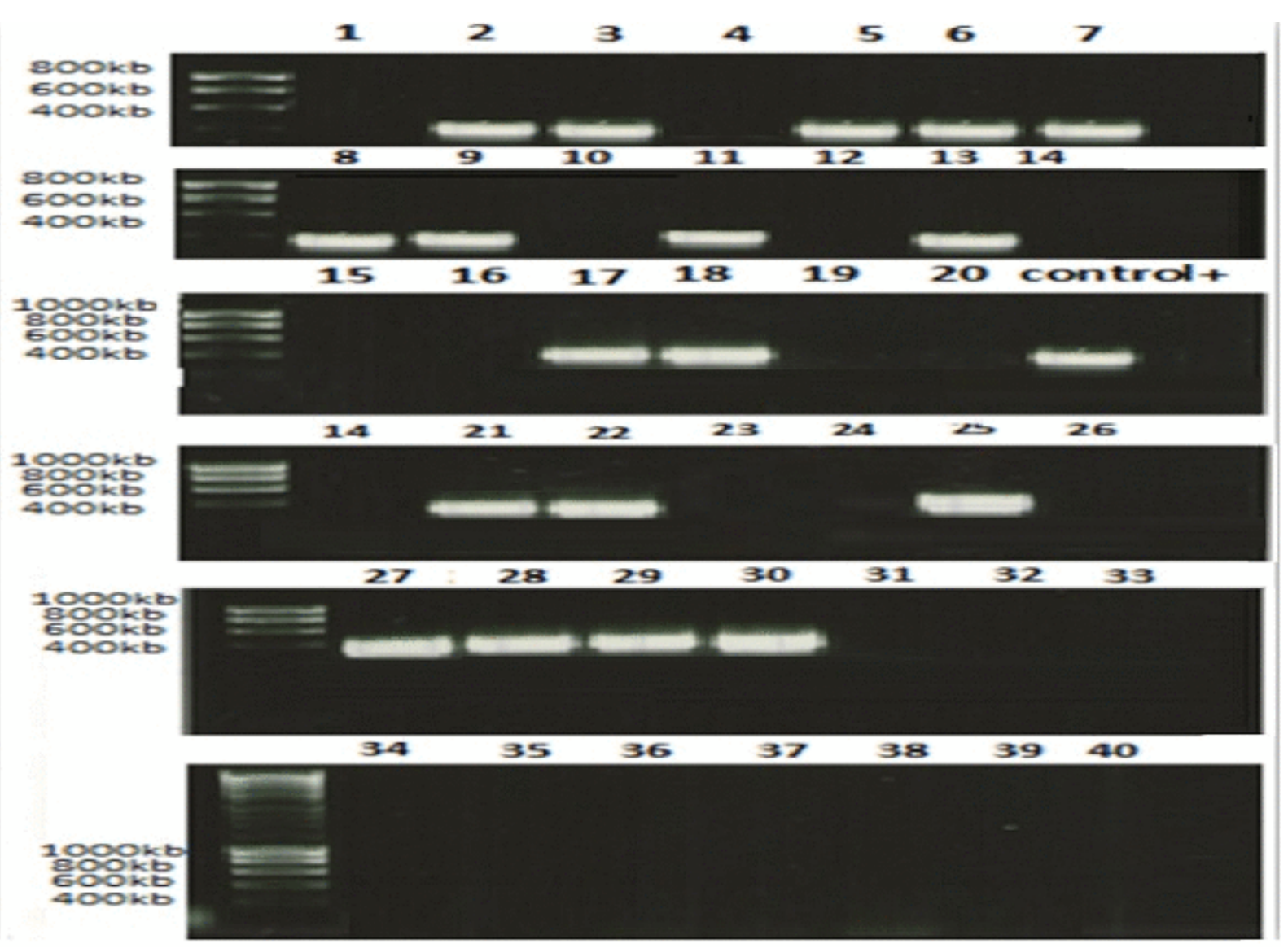

Figure 1: The results of the PCR-screening of SesC genes in clinical isolates; Sample's numbers 1 (S. haemolyticus), 2 (S. epidermidis), 3 (S. epidermidis), 4 (S. epidermidis), 5 (S. epidermidis), 6 (S. epidermidis), 7 (S. epidermidis), 8 (S. epidermidis), 9 (S. epidermidis), 10 (S. haemolyticus), 11 (S. epidermidis), 12 (S. lugdunensis), 13 (S. epidermidis), 14 (S. haemolyticus), 15 (S. hominis), 16 (S. warneri), 17 (S. epidermidis), 18 (S. epidermidis), 19 (S. haemolyticus), 20 (S. haemolyticus), 14-2 (S. epidermidis), 21 (S. epidermidis), 22 (S. epidermidis), 23 (S. hominis), 24 (S. haemolyticus), 25 (S. epidermidis), 26 (S. haemolyticus), 27 (S. epidermidis), 28 (S. epidermidis), 29 (S. epidermidis), 30 (S epidermidis), 31 (S. haemolyticus), 32 (S. haemolyticus), 33 (S. haemolyticus), 34 (S. hominis), 35 (S. warneri), 36 (S. pasteuri), 37 (S. warneri), 38 (S. haemolyticus), 39 (S. warneri), 40 (S. aureus), positive control was RP62A S. epidermidis and negative control was Distilled water.

\section{In vitro biofilm formation}

In the preliminary assessment on biofilm formation of isolates in the plate was identified that, among the $20 \mathrm{~S}$. epidermidis strains, 3 samples (samples 13, 28, 29) did not have the ability to form biofilm or were very weak, so they were not used in the further experiments (Table 2). 
Citation: Fard SS, Amin M, Khodaparast L, Khodaparast L, Choghakabodi PM, et al. (2015) Detection of Biofilm Phenotype of Isolated Staphylococcus epidermidis from Respiratory Catheters of Hospitalized Patients and Evaluation the Effect of Antibodies against SesC Protein on Biofilm Formation. Clin Microbiol 4: 221. doi:10.4172/2327-5073.1000221

Page 4 of 6

\begin{tabular}{|c|c|c|c|c|c|c|c|}
\hline Sample number & Species & $\operatorname{ses} C$ & Biofilm-TSB & B-Glu & B-NaCl & SM & PK \\
\hline 2 & S. epidermidis & $P$ & $P$ & $P$ & $P$ & d & nd \\
\hline 3 & S. epidermidis & $P$ & $P$ & $P$ & $P$ & $d$ & nd \\
\hline 4 & S. epidermidis & $P$ & $\mathrm{~N}$ & $P$ & $\mathrm{~N}$ & nd & d \\
\hline 5 & S. epidermidis & $P$ & $\mathrm{~N}$ & $P$ & $N$ & nd & $d$ \\
\hline 6 & S. epidermidis & $\mathrm{P}$ & $\mathrm{N}$ & $\mathrm{P}$ & $\mathrm{N}$ & nd & nd \\
\hline 7 & S. epidermidis & $P$ & $P$ & $P$ & $N$ & nd & $d$ \\
\hline 8 & S. epidermidis & $P$ & $P$ & $P$ & $P$ & d & nd \\
\hline 9 & S. epidermidis & $P$ & $P$ & $P$ & $N$ & nd & nd \\
\hline 11 & S. epidermidis & $P$ & $P$ & $P$ & $P$ & $d$ & nd \\
\hline 13 & S. epidermidis & $P$ & N & $N$ & $N$ & - & - \\
\hline 14 & S. epidermidis & $P$ & $\mathrm{~N}$ & $P$ & $N$ & $\mathrm{nd}$ & $d$ \\
\hline 17 & S. epidermidis & $P$ & $\mathrm{~N}$ & $P$ & $N$ & $\mathrm{nd}$ & $d$ \\
\hline 18 & S. epidermidis & $\mathrm{P}$ & $\mathrm{N}$ & $P$ & $N$ & nd & $d$ \\
\hline 21 & S. epidermidis & $P$ & $\mathrm{~N}$ & $P$ & $\mathrm{~N}$ & nd & $d$ \\
\hline 22 & S. epidermidis & $P$ & $\mathrm{~N}$ & $P$ & $\mathrm{~N}$ & nd & $d$ \\
\hline 25 & S. epidermidis & $\mathrm{P}$ & $P$ & $P$ & $P$ & nd & nd \\
\hline 27 & S. epidermidis & $P$ & ${ }^{*} \mathrm{P}$ & ${ }^{*} \mathrm{P}$ & ${ }^{*} \mathrm{P}$ & $d$ & nd \\
\hline 28 & S. epidermidis & $\mathrm{P}$ & $\mathrm{N}$ & N & $\mathrm{N}$ & - & - \\
\hline 29 & S. epidermidis & $P$ & $\mathrm{~N}$ & $P$ & $N$ & - & _- \\
\hline 30 & S. epidermidis & $\mathrm{P}$ & $P$ & $P$ & $P$ & $d$ & nd \\
\hline
\end{tabular}

Table 2: The results of the effect of different stimulants on biofilm formation of isolates and biofilm phenotype in isolates.

\section{Determination of biofilm-forming types of bacteria}

Six isolates $(30 \%)$ produced dissolvable polysaccharide intercellular adhesin (PIA)-dependent biofilms in SM after growth in TSB with $\mathrm{NaCl}$ and eight isolates (40\%) produced dissolvable protein-dependent biofilms in PK after growth in TSB with glucose (Table 1). At this stage also were excluded a number of cases that were weak in biofilm formation and or that had a large variation (samples 4, 5, 7, 9, 17, 18, 21).

\section{Inhibitory effect of anti-SesC antibodies on biofilm formation}

In this test that was conducted to evaluate the effect of specific antiSesC antibodies ( $a \mathrm{Ses} C$-IgGs) on biofilms, were used only 10 samples that were screened from the previous experiments (samples $2,3,14,22$, $6,11,30,27,8,25)$.

\section{Analysis of the results using statistical methods}

After reviewing the results by TWO-Way ANOVA analysis and Evaluation of P-value (PV: 0.0001), it was observed that anti-SesC antibodies had significant inhibitory effect on biofilm formation in $40 \%$ of cases $(\mathrm{P}<0.05)$ in particular on protein-dependent biofilms (Figure 2) $[6,10]$.

\section{Discussion}

In the present study, the emphasis is on the importance of biofilm formation in pathogenesis of Staphylococcus epidermidis, particularly role of SesC protein on biofilm formation. $S$. epidermidis is responsible for the vast majority of nosocomial catheter related blood stream infections (CRBSI) in the United States [12]. Most significantly, $S$. epidermidis is the leading organism isolated from foreign material related infections (FMRI) [13] such as infected prosthetic joints, central venous catheters (CVC), cerebrospinal fluid shunts, intracardiac devices, artificial heart valves, and vascular grafts $[6,14]$. Although many factors are involved in the pathogenesis of $S$. epidermidis, but the incidence of infection due to the use of medical devices is dependent on the ability of bacteria to produce a hard multilayer structure called "biofilm" on the surface of Medical devices used to treat [2]. In 2005, a study by Bowden and et.al carried out, that with reviewing of 116 clinical isolates of $S$. epidermidis, were able to identify $\mathrm{Ses} C$ genes in these isolates and subsequently introduced the product 
Citation: Fard SS, Amin M, Khodaparast L, Khodaparast L, Choghakabodi PM, et al. (2015) Detection of Biofilm Phenotype of Isolated Staphylococcus epidermidis from Respiratory Catheters of Hospitalized Patients and Evaluation the Effect of Antibodies against SesC Protein on Biofilm Formation. Clin Microbiol 4: 221. doi:10.4172/2327-5073.1000221

Page 5 of 6

of this gene as an important factor in the pathogenesis of these bacteria

[15].

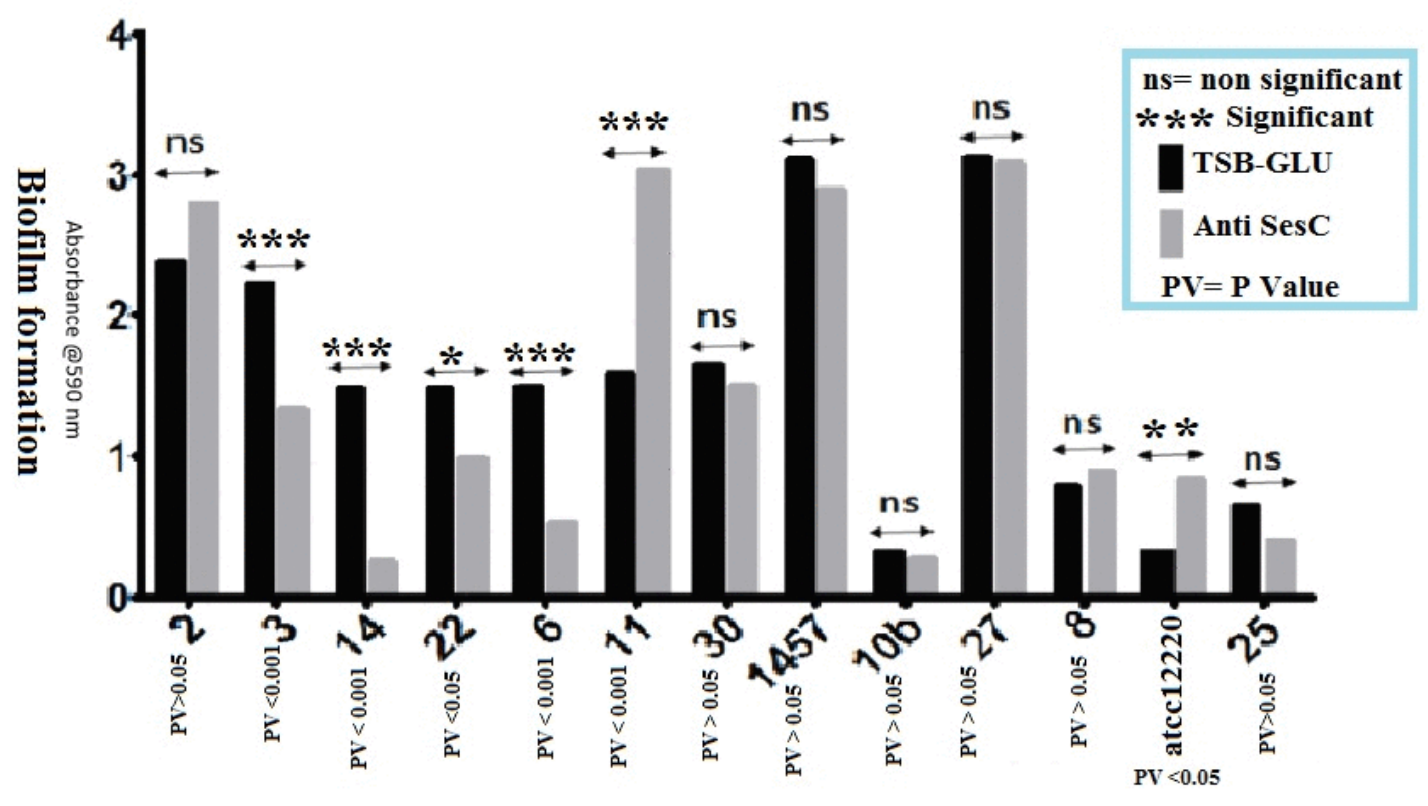

Different Strains

Figure 2: The results Analysis of by using two-way ANOVA analysis; atcc12220 was negative control and $10 \mathrm{~b}$ and 1457 were positive control. Anti-SesC antibodies had significant inhibitory effect on biofilm formation in $40 \%$ of cases \{samples $3,14,22,6\}(\mathrm{P}<0.05)$.

In a series of studies that were carried out by Shahroei et al. [6] in order to assess the SesC protein as an appropriate vaccine candidate against biofilm formation by $S$. epidermidis, it was observed that the $\mathrm{Ses} C$-antibodies have shown a significant inhibitory effect on the biofilm formation of bacteria in vitro, and also these SesC-antibodies have shown significant inhibitory effects on bacterial colonization and infection in a test on an experimental model of mouse jugular vein catheter infection that includes biofilms and organ infections [10]. For this reason, in this study we also investigated the presence of sesC gene in the strains and the effect of anti-SesC on their biofilm. The most important aim of this study is detection of biofilm phenotype of isolated $S$. epidermidis from respiratory catheters of hospitalized patients and evaluation of the effect of antibodies against SesC protein on biofilm formation. According to the recent studies the formed biofilm by $S$. epidermidis is mediated by polysaccharide, protein factors and in some cases by eDNA [16]. Many surface proteins in staphylococci have been studied to use as a target to cope with biofilm formation [17]. In this study, we've used of PCR screening of SesC genes in clinical isolates. As it was previously reported, ses $\mathrm{C}$ is more strongly expressed in biofilm-associated cells in $S$. epidermidis strains [10]. we also used of semi-quantitative adherence assay in 96-well polystyrene microtiter plates (BD Biosciences, Heidelberg, Germany) as previously described [9] similar to the Shahroei et al. [6] study; After determining the bacteria produced biofilm, the effect of SM and PK on biofilm formation was evaluated in order to determine the biofilm phenotype. PIA-dependent biofilms dissolved in SM after growth in TSB with $\mathrm{NaCl}$ but protein-dependent biofilms dissolved in PK after growth in TSB with glucose. In this study, $S$. epidermidis strains had the highest frequency among all the isolated CoNS strains. Most of these strains were able to form biofilm. A total of $70 \%$ strains were able to form biofilm (30\% PIA-dependent biofilms and $40 \%$ proteindependent biofilms). Two-way ANOVA analysis indicated that antiSesC antibodies had significant inhibitory effect on biofilm formation only in $40 \%$ of cases $(\mathrm{P}<0.05)$. In conclusion, our work has underlined the necessity to further investigation for finding a way to prevent of infection by these bacteria. Although, the exact role of SesC in biofilm formation still is unknown but our findings display the importance of SesC protein on developing of biofilm formation of respiratory catheters. On the other hand, it is recommended that doctors should be aware of antibiogram results to treat infections caused by these strains. Since all $S$. epidermidis strains $(\mathrm{n}=20)$ resistant against to Benzyl-Penicillin and susceptible to Vancomycin, Tigecycline and Nitrofurantoin antibiotics, it is suggested that doctors should use of these antibiotics to treat infections caused by these strains.

\section{Acknowledgments}

This work was supported by the Arvand International Division, Ahvaz Jundishapur University of Medical Sciences, Iran.

\section{References}

1. Otto M (2009) Staphylococcus epidermidis--the 'accidental' pathogen. Nat Rev Microbiol 7: 555-567.

2. Hidron AI, Edwards JR, Patel J, Horan TC, Sievert DM, et al. (2008) NHSN annual update: antimicrobial-resistant pathogens associated with healthcare-associated infections: annual summary of data reported to the National Healthcare Safety Network at the Centers for Disease Control and Prevention, 2006-2007. Infect Control Hosp Epidemiol 29: 996-1011. 
Citation: Fard SS, Amin M, Khodaparast L, Khodaparast L, Choghakabodi PM, et al. (2015) Detection of Biofilm Phenotype of Isolated Staphylococcus epidermidis from Respiratory Catheters of Hospitalized Patients and Evaluation the Effect of Antibodies against SesC Protein on Biofilm Formation. Clin Microbiol 4: 221. doi:10.4172/2327-5073.1000221

Page 6 of 6

3. Geipel U, Herrmann M (2005) [The infected implant: bacteriology]. Unfallchirurg 108: 961-975.

4. Mack D, Horstkotte MA, Rohde H, Knobloch JKM (2006) Coagulasenegative staphylococci, in Biofilms, Infection, and Antimicrobial Therapy. CRC Press, Boca Raton, USA.

5. Monk IR, Shah IM, Xu M, Tan MW, Foster TJ (2012) Transforming the untransformable: application of direct transformation to manipulate genetically Staphylococcus aureus and Staphylococcus epidermidis. MBio 3: e00277.

6. Shahrooei M, Hira V, Khodaparast L, Khodaparast L, Stijlemans B, et al. (2012) Vaccination with SesC Decreases Staphylococcus epidermidis Biofilm Formation. Infect Immun 80: 3660-3668.

7. Rogers KL, Fey PD, Rupp ME (2009) Coagulase-negative staphylococcal infections. Infect Dis Clin North Am 23: 73-98.

8. Yates JR 3rd (2000) Mass spectrometry. From genomics to proteomics Trends Genet 16: 5-8.

9. Christensen GD, Simpson WA, Younger JJ, Baddour LM, Barrett FF, et al. (1985) Adherence of coagulase-negative staphylococci to plastic tissue culture plates: a quantitative model for the adherence of staphylococci to medical devices. J Clin Microbiol 22: 996-1006.

10. Shahrooei M, Hira V, Stijlemans B, Merckx R, Hermans PW, et al. (2009) Inhibition of Staphylococcus epidermidis biofilm formation by rabbit polyclonal antibodies against the $\mathrm{Ses} \mathrm{C}$ protein. Infect Immun 77 3670-3678.
11. McCann MT, Gilmore BF, Gorman SP (2008) Staphylococcus epidermidis device-related infections: pathogenesis and clinical management. J Pharm Pharmacol 60: 1551-1571.

12. Hussain $M$, Herrmann $M$, von Eiff $C$, Perdreau-Remington F, Peters $G$ (1997) A 140-kilodalton extracellular protein is essential for the accumulation of Staphylococcus epidermidis strains on surfaces. Infect Immun 65: 519-524.

13. Gilbert P, Das J, Foley I (1997) Biofilm susceptibility to antimicrobials. Adv Dent Res 11: 160-167.

14. Mack D, Davies AP, Harris LG, Rohde H, Horstkotte MA, et al. (2007) Microbial interactions in Staphylococcus epidermidis biofilms. Anal Bioanal Chem 387: 399-408.

15. Bowden MG, Chen W, Singvall J, Xu Y, Peacock SJ, et al. (2005) Identification and preliminary characterization of cell-wall-anchored proteins of Staphylococcus epidermidis. Microbiology 151: 1453-1464.

16. Otto M (2012) Molecular basis of Staphylococcus epidermidis infections. Semin Immunopathol 34: 201-214.

17. Arrecubieta C, Toba FA, von Bayern M, Akashi H, Deng MC, et al. (2009) SdrF, a Staphylococcus epidermidis surface protein, contributes to the initiation of ventricular assist device driveline-related infections. PLoS Pathog 5: e1000411. 